\title{
Thromboelastography parameters in diagnosing periprosthetic joint infection and predicting reimplantation timing
}

Tao Yuan ${ }^{1}$, Yi Wang ${ }^{1}$ and Shui Sun ${ }^{1,2^{*}}$ (i)

\begin{abstract}
Background: Coagulation-related biomarkers are drawing new attention in the diagnosis of periprosthetic joint infection (PJI). The thromboelastography (TEG) assay provides a comprehensive assessment of blood coagulation; therefore, it could be a promising test for PJI. This study aims to assess the value of TEG in diagnosing PJI and to determine the clinical significance of TEG in analysing reimplantation timing for second-stage revision.

Methods: From October 2017 to September 2020, 62 patients who underwent revision arthroplasty were prospectively included. PJI was defined by the 2011 Musculoskeletal Infection Society criteria, in which 23 patients were diagnosed with PJI (Group A), and the remaining 39 patients were included as having aseptic loosening (Group B). In group A, 17 patients completed a two-stage revision in our centre. C-reactive protein (CRP), erythrocyte sedimentation rate (ESR), D-dimer, and TEG parameters (clotting time, a-angle, MA [maximum amplitude], amplitude at $30 \mathrm{~min}$, and thrombodynamic potential index) were measured preoperatively in all included patients. In addition, receiver operating characteristic curves were used to evaluate the diagnostic value of these biomarkers.

Results: ESR (area under curve [AUC], 0.953; sensitivity, 81.82; specificity, 94.87) performed best for PJI diagnosis, followed by MA (AUC, 0.895; sensitivity, 82.61; specificity, 97.44) and CRP (AUC, 0.893; sensitivity, 82.61; specificity, 94.74). When these biomarkers were combined in pairs, the diagnostic value improved compared with any individual biomarker. The overall success rate of the two-stage revision was 100\%. Furthermore, ESR and MA were valuable in determining the time of reimplantation, and their values all decreased below the cut-off values before reimplantation.
\end{abstract}

Conclusion: TEG could be a promising test in assisting PJI diagnosis, and a useful tool in judging the proper timing of reimplantation.

Keywords: Thromboelastography, Periprosthetic joint infection, Coagulation-related biomarkers, Two-stage revision

\footnotetext{
* Correspondence: sunshui1965@163.com

1 Department of Joint Surgery, Shandong Provincial Hospital, Cheeloo College

of Medicine, Shandong University, Jinan 250012, Shandong, China

${ }^{2}$ Department of Joint Surgery, Shandong Provincial Hospital Affiliated to

Shandong First Medical University, Jinan, Shandong, China
}

(C) The Author(s). 2021 Open Access This article is licensed under a Creative Commons Attribution 4.0 International License, which permits use, sharing, adaptation, distribution and reproduction in any medium or format, as long as you give appropriate credit to the original author(s) and the source, provide a link to the Creative Commons licence, and indicate if changes were made. The images or other third party material in this article are included in the article's Creative Commons. licence, unless indicated otherwise in a credit line to the material. If material is not included in the article's Creative Commons licence and your intended use is not permitted by statutory regulation or exceeds the permitted use, you will need to obtain permission directly from the copyright holder. To view a copy of this licence, visit http://creativecommons.org/licenses/by/4.0/. The Creative Commons Public Domain Dedication waiver (http://creativecommons.org/publicdomain/zero/1.0/) applies to the data made available in this article, unless otherwise stated in a credit line to the data. 


\section{Background}

Periprosthetic joint infection (PJI), one of the most troublesome complications of total hip or knee arthroplasty, exacerbates the burden on the individual and health care system [1-4]. As the number of surgeries surged yearly, the total number of PJI patients increased [5]. However, the diagnosis and treatment of PJI remain challenging for clinicians.

Current diagnostic methods of PJI include serological testing, synovial fluid testing, and intraoperative histological pathology [2, 5]. However, although the diagnostic criteria are well defined, no gold standard has yet been established $[4,6]$. Similarly, treatment of PJI is difficult for clinicians because there are no widely accepted criteria $[7,8]$. The two-stage revision is currently the standard procedure for PJI, but the proper time to perform the second-stage revision is still debatable [9].

As recent literature revealed the close correlations between the coagulation cascade and infection course, coagulation-related biomarkers are gaining attention. Some biomarkers, such as D-dimer and fibrinogen (Fib), have been proven promising for PJI diagnosis [10-13] and determining the reimplantation timing [10, 14].

Thromboelastography (TEG) is a routine coagulation test that assesses the whole process of clotting over time in the body [15] and provides a full-scale evaluation of clot formation, elasticity, and duration. Additionally, various coagulation elements are measured as follows [16]. The clotting time ( $\mathrm{K}$ value) reflects the rate of blood clot formation and is an indicator of fibrinogen function [17]. The $\alpha$-angle (angle) represents the clot growth rate, while the maximum amplitude (MA) is the maximum clot amplitude [18]. The amplitude at $30 \mathrm{~min}$ (A30) measures clot strength at $30 \mathrm{~min}$ after MA [19], and thrombodynamic potential index (TPI) was derived from the MA and $K$ values [20].

Moreover, TEG yields information about all phases of coagulation and provides further information on standard coagulation tests $[16,21]$. Numerous studies have proved that TEG is useful in evaluating coagulation status, predicting bleeding in patients with severe sepsis, monitoring haemostasis during cardiac surgery and liver transplant procedures, etc. $[15,21,22]$. However, no study has reported its value in diagnosing PJI and guiding the timing of reimplantation for the second-stage revision.

Therefore, this study aims to investigate (1) the value of TEG in distinguishing PJI from aseptic loosening and (2) the ability of TEG parameters to guide the proper time for the second-stage revision. Furthermore, the measured TEG parameters were compared with the ESR, CRP, and D-dimer levels.

\section{Methods}

We conducted this retrospective study including all revision total hip and total knee arthroplasties performed in our hospital from October 2017 to September 2020, under the ethical approval of the institutional review board of our hospital. Among the 145 patient records acquired, 61 patients were diagnosed with PJI according to the Musculoskeletal Infection Society criteria [23], and 84 patients were diagnosed with aseptic loosening. Patients were excluded if at least one of the following are present: (1) lack of needed data, (2) recent use of anticoagulants, (3) presence of inflammatory arthritis such as rheumatoid arthritis and ankylosing spondylitis, (4) blood diseases such as thrombocytopenic purpura, (5) formation of deep vein thrombosis of the lower limbs, (6) liver diseases, (7) malignancy, and (8) infection of other tissues or organs.

Finally, 62 patients who underwent revision arthroplasty were included in this study: 23 in group A (treated for PJI) and 39 in group B (treated for aseptic loosening). A total of 83 patients were excluded due to lack of needed data $(n=55)$, deep vein thrombosis in the lower limbs $(n=24)$, recent use of oral warfarin due to coronary stent implantation $(n=2)$, urinary tract infection $(n=1)$, and rheumatoid arthritis $(n=1)$.

The patients' fasting venous blood samples were collected routinely on the second day of admission and sent to the clinical laboratory of our hospital for blood examination, including routine blood examination, conventional coagulation tests, and TEG. The test results were acquired approximately $30 \mathrm{~min}$ after blood collection. Moreover, at least 3 tissue culture specimens (including joint fluid) were obtained when the participants underwent revision arthroplasty, and these samples were cultured for 7-14 days. Group B underwent a one-stage revision. Meanwhile, the two-stage revision for group A consisted of the following procedures: 1) The first-stage revision involved removal of the former prosthesis, supervened with implantation of antibiotic-loaded cement spacers $(4 \mathrm{~g}$ vancomycin in $160 \mathrm{~g}$ gentamicin-containing bone cement). 2) At least 3 months after the first stage of treatment, surgeons decided whether to implant a new prosthesis or continue antibiotic protocols based on clinical symptoms and laboratory parameters. Aseptic patients were prescribed oral rivaroxaban $10 \mathrm{mg}$ daily for 35 days for thromboprophylaxis, and PJI patients followed the same order after each staged surgery. All included patients were regularly followed up at 1 month, 3 months, 6 months, 1 year, and then each year after discharge. Functional outcomes, complications, and the reasons for any reoperation were recorded. According to Delphi-based consensus, success of reimplantation was defined by (1) control of infection, as characterised by a healed wound without fistula, drainage, or pain; (2) no 
subsequent surgical intervention for infection after reimplantation surgery; and (3) no occurrence of PJI-related mortality $[24,25]$.

\section{Statistical analysis}

Statistical analysis was performed using IBM SPSS Statistics for Windows (version 26; IBM Corporation, Armonk, NY, USA), and statistical significance was set at $p<0.05$. Independent sample $\mathrm{t}$-tests were used for data conforming to normal distributions. In contrast, the Mann-Whitney U tests were used for data not conforming to normal distributions, and categorical variables were summarised using chi-squared tests. Receiver operating characteristic (ROC) curves were drawn using SPSS. The following parameters were calculated in each test: area under the curves (AUCs), sensitivity, specificity, positive predictive value (PPV), negative predictive value (NPV), positive likelihood ratio (+LR), and negative likelihood ratio (-LR). Moreover, we further studied the diagnostic value of the different combinations of mentioned parameters in pairs. The Youden index was used to determine the optimal cut-off value of these biomarkers for the diagnosis of PJI. Furthermore, a scatterplot was drawn using GraphPad Prism (version 8.0.2; GraphPad Software)8.

Table 1 Demographics of the Study Groups

\begin{tabular}{|c|c|c|c|}
\hline Demographics & Group A $(n=23)$ & Group B $(n=39)$ & $p$ Value \\
\hline Age $(y)^{a}$ & $64.13 \pm 9.10$ & $64.56 \pm 10.88$ & .873 \\
\hline $\operatorname{Sex}^{\mathrm{b}}$ & & & .424 \\
\hline Male & $10(43.48 \%)$ & $13(33.33 \%)$ & \\
\hline Female & $13(56.52 \%)$ & $26(66.67 \%)$ & \\
\hline $\operatorname{BMI}\left(\mathrm{kg} / \mathrm{m}^{2}\right)^{\mathrm{a}}$ & $26.16 \pm 2.83$ & $25.55 \pm 3.61$ & .493 \\
\hline Involved joint ${ }^{\mathrm{b}}$ & & & $<0.01$ \\
\hline Hip & 7 (30.43\%) & $34(87.18 \%)$ & \\
\hline Knee & $16(69.57 \%)$ & $5(12.82 \%)$ & \\
\hline \multicolumn{4}{|c|}{ Serum inflammatory and fibrinolytic markers } \\
\hline $\mathrm{CRP}(\mathrm{mg} / \mathrm{L})$ & $45.71 \pm 54.50$ & $3.57 \pm 4.62$ & $<.05$ \\
\hline $\operatorname{ESR}(\mathrm{mm} / \mathrm{h})$ & $57.59 \pm 27.97$ & $14.59 \pm 10.33$ & $<.001$ \\
\hline D-dimer (mg/L) & $1.72 \pm 1.22$ & $1.06 \pm 1.23$ & $<.001$ \\
\hline$K(\min )$ & $1.26 \pm 0.77$ & $1.56 \pm 0.41$ & $<.001$ \\
\hline Angle $\left(^{\circ}\right)$ & $72.56 \pm 7.38$ & $67.80 \pm 6.13$ & $<.001$ \\
\hline $\mathrm{MA}(\mathrm{mm})$ & $71.23 \pm 5.62$ & $62.83 \pm 4.03$ & $<.001$ \\
\hline $\mathrm{A} 30(\mathrm{~mm})$ & $70.85 \pm 5.79$ & $62.36 \pm 4.16$ & $<.001$ \\
\hline TPI(/sec) & $126.28 \pm 54.46$ & $60.30 \pm 26.24$ & $<.001$ \\
\hline
\end{tabular}

$B M I$ body mass index, $S D$ standard deviation, $C R P$ C-reactive protein, ESR erythrocyte sedimentation rate, $K$ clotting time, Angle a-angle, $M A$ maximum amplitude, $A 30$ amplitude at $30 \mathrm{~min}$, TPI thrombodynamic potential index

${ }^{a}$ The values are expressed as the mean \pm SD

${ }^{b}$ The values are expressed as the numbers of patients, with the percentage in parentheses

\section{Results}

The demographic characteristics of each group are shown in Table 1 . There were no statistically significant differences between baselines of the two study groups, except for involved joints. Particularly, the hip joint accounted for $87.18 \%$ in group B and $30.43 \%$ in group A, which was statistically significant $(p<0.01)$.

We observed significant differences in tested markers between Groups A and B $(p<.001)$, as shown in Table 1 . The median values of CRP, ESR, D-dimer, and TEG parameters (Angle, MA, A30, TPI) in group A were significantly higher than those in group $\mathrm{B}(p<.001)$. In contrast, the median $\mathrm{K}$ value was lower in group $\mathrm{A}$ $(p<.001)$.

To evaluate and compare the diagnostic value of the tested markers, ROC curves of each inflammatory and fibrinolytic marker are illustrated in Fig. 1, while the AUC of each ROC curve was calculated (Table 2). The biomarkers' optimal cut-off values for the diagnosis of PJI are shown in Table 2. The AUCs for CRP, ESR, and D-dimer were 0.893 (95\% confidence interval [CI], 0.787-0.958), 0.953 (95\% CI, 0.866-0.991), and 0.717 (95\% CI, 0.588-0.824), respectively. The AUCs of TEG parameters ranged from 0.800 (K value, 95\% CI, 0.680 0.891 ) to 0.895 (MA, 95\% CI, 0.790-0.958). Among all tested biomarkers, ESR had the highest AUC, while Ddimer had the lowest AUC. The AUC of MA ranked first among the TEG parameters, followed by A30, TPI, Angle, and $\mathrm{K}$ value. Although the AUC was lower than that of ESR, MA achieved a better sensitivity and specificity.

The diagnostic values of the different combinations of tested parameters in pairs are shown in Table 3. The combination of TEG parameters and CRP/ESR led to improved AUC, sensitivity, and specificity, except for $\mathrm{ESR}+\mathrm{MA}$ and ESR + A30. In addition, CRP + A30 achieved an obvious boost in the diagnosis value.

The culture results of 23 patients with PJI are listed in Table 4, wherein 19 (82.6\%) patients had positive results. Gram-positive bacteria, particularly $S$. aureus, are the most common pathogens observed. According to Table 5, the values of CRP, ESR, MA, A30, and TPI in Fungi group were less than those of the other three groups (G+ group, G- group and negative group).

Data from 17 patients who underwent two-stage revision surgery were available in the history database management system. The other six patients could not be readmitted for the second-stage surgery due to short intervals from the first-stage surgery (less than 3 months). According to Delphi-based consensus, no patients showed a failure of reimplantation during the follow-up period (17.29 \pm 8.29 (range, 3-28) months). However, 2 of 17 patients had poor knee function with limited range of motion. On comparing the tested biomarkers between 

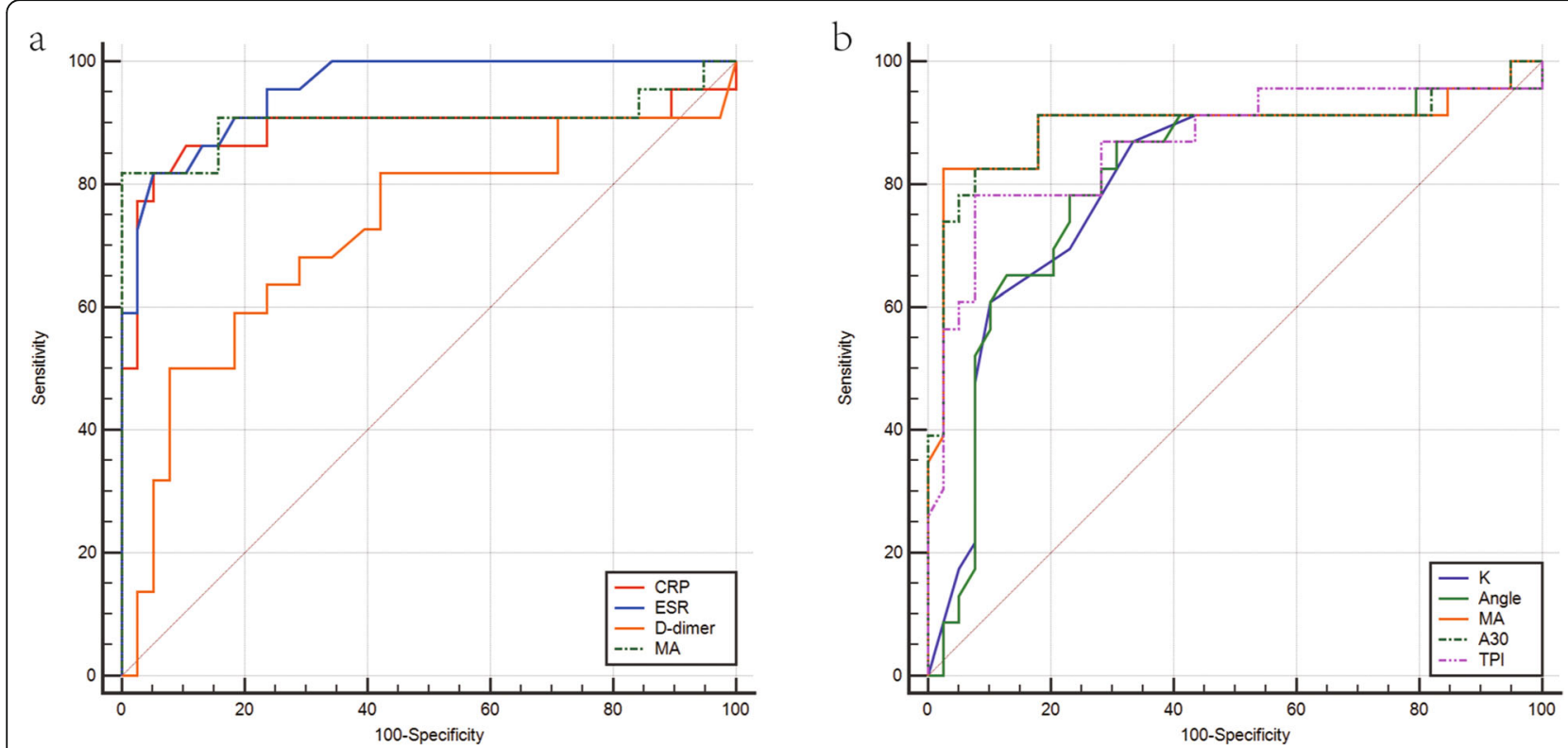

Fig. 1 The comparison of ROC curves. ROC, receiver operating characteristic curve. CRP, C-reactive protein; ESR, erythrocyte sedimentation rate; MA, maximum amplitude; $\mathrm{K}$, clotting time; Angle, a-angle; A30, amplitude at $30 \mathrm{~min}$; TPI, thrombodynamic potential index

stage 1 (the stage before spacer insertion) and stage 2 (the stage after re-admission for the second-stage surgery) of PJI patients, all tested markers except for Ddimer $(p=0.059)$ were found statistically significant. Furthermore, the numerical values of ESR and MA decreased below the cut-off value during stage 2 (Fig. 2) when each patient's parameters were compared with corresponding cut-off values.

\section{Discussions}

Many studies have revealed a close association between coagulation and infection. Endotoxins or components of the bacterial cell wall were reported to trigger changes in coagulation through tissue factor (TF) released by a variety of cells, such as vascular endothelial cells and monocytes, and maintain TF at high levels by stimulating the release of cytokines, such as interleukins and tumour necrosis factor $\alpha[21,26,27]$. Correspondingly, coagulation-related biomarkers have recently been proven to be valuable in PJI diagnosis. D-dimer has been adopted as a minor criterion in the 2018 International Consensus Meeting criteria for PJI, but it has raised a huge controversy. Yan et al. [28] conducted a metaanalysis and observed that D-dimer is an effective serum biomarker for PJI diagnosis in patients without a history of hypercoagulation or inflammatory arthritis. In contrast, Tejbir et al. [2] and Rui Li et al. [29] reported the poor diagnostic value of D-dimer for PJI. Meanwhile,

Table 2 The Diagnostic Value of Inflammatory and Fibrinolytic Markers

\begin{tabular}{|c|c|c|c|c|c|c|c|c|c|}
\hline Markers & AUC & Optimal cutoff & Youden index & SEN (\%) & SPE (\%) & PPV (\%) & NPV (\%) & $+\mathrm{LR}$ & $\overline{-L R}$ \\
\hline $\mathrm{CRP}(\mathrm{mg} / \mathrm{L})$ & 0.893 & $>8.8$ & 0.7735 & 82.61 & 94.74 & 90.5 & 90.0 & 15.70 & 0.18 \\
\hline $\mathrm{ESR}(\mathrm{mm} / \mathrm{h})$ & 0.953 & $>34.0$ & 0.7669 & 81.82 & 94.87 & 90.0 & 90.2 & 15.95 & 0.19 \\
\hline D-dimer (mg/L) & 0.717 & $>1.7$ & 0.4013 & 47.83 & 92.31 & 78.6 & 75.0 & 6.22 & 0.57 \\
\hline $\mathrm{K}(\min )$ & 0.800 & $<1.3$ & 0.5362 & 86.96 & 66.67 & 60.6 & 89.7 & 2.61 & 0.20 \\
\hline Angle $\left(^{\circ}\right)$ & 0.803 & $>70.7$ & 0.5619 & 86.96 & 69.23 & 62.5 & 90.0 & 2.83 & 0.19 \\
\hline $\mathrm{MA}(\mathrm{mm})$ & 0.895 & $>68.1$ & 0.8004 & 82.61 & 97.44 & 95.0 & 90.5 & 32.22 & 0.18 \\
\hline $\mathrm{A} 30(\mathrm{~mm})$ & 0.893 & $>66.5$ & 0.7492 & 82.61 & 92.31 & 86.4 & 90.0 & 10.74 & 0.19 \\
\hline TPI(/sec) & 0.867 & $>84.4$ & 0.7057 & 78.26 & 92.31 & 85.7 & 87.8 & 10.17 & 0.24 \\
\hline
\end{tabular}

AUC area under the curve, SEN sensitivity, SPE specificity, $P P V$ positive predictive value, NPV negative predictive value, $+L R$ positive likelihood ratio, $-L R$ negative likelihood ratio, CRP C-reactive protein, ESR erythrocyte sedimentation rate, $K$ clotting time, Angle a-angle, $M A$ maximum amplitude, $A 30$ amplitude at 30 min, $T P I$ thrombodynamic potential index 
Table 3 The Diagnostic Value of Combined Inflammatory and Fibrinolytic Markers

\begin{tabular}{|c|c|c|c|c|c|c|c|}
\hline Markers & AUC & SEN (\%) & SPE (\%) & PPV (\%) & NPV (\%) & $+\mathrm{LR}$ & $-\mathrm{LR}$ \\
\hline $\mathrm{CRP}+\mathrm{ESR}$ & 0.980 & 100.00 & 89.47 & 84.6 & 100.0 & 9.50 & 0.000 \\
\hline $\mathrm{CRP}+\mathrm{K}$ & 0.943 & 91.30 & 94.74 & 91.3 & 94.7 & 17.35 & 0.092 \\
\hline CRP + Angle & 0.946 & 91.30 & 94.74 & 91.3 & 94.7 & 17.35 & 0.092 \\
\hline $\mathrm{CRP}+\mathrm{MA}$ & 0.995 & 100.00 & 94.74 & 92.0 & 100.0 & 19.00 & 0.000 \\
\hline$C R P+A 30$ & 0.999 & 100.00 & 97.37 & 95.8 & 100.0 & 38.00 & 0.000 \\
\hline$C R P+T P I$ & 0.968 & 91.30 & 97.37 & 95.5 & 94.9 & 34.70 & 0.089 \\
\hline$E S R+K$ & 0.958 & 86.36 & 94.87 & 90.5 & 92.5 & 16.84 & 0.140 \\
\hline ESR + Angle & 0.953 & 86.36 & 94.87 & 90.5 & 92.5 & 16.84 & 0.140 \\
\hline $\mathrm{ESR}+\mathrm{MA}$ & 0.948 & 90.91 & 89.74 & 83.3 & 94.6 & 8.86 & 0.100 \\
\hline $\mathrm{ESR}+\mathrm{A} 30$ & 0.948 & 81.82 & 97.44 & 94.7 & 90.5 & 31.91 & 0.190 \\
\hline $\mathrm{ESR}+\mathrm{TPI}$ & 0.955 & 81.82 & 97.44 & 94.7 & 90.5 & 31.91 & 0.190 \\
\hline
\end{tabular}

AUC area under the curve, SEN sensitivity, SPE specificity, $P P V$ positive predictive value, NPV negative predictive value, $+L R$ positive likelihood ratio, $-L R$ negative likelihood ratio, $C R P$ C-reactive protein, $K$ clotting time, Angle a-angle, MA maximum amplitude, $A 30$ amplitude at 30 min, TPI thrombodynamic potential index, ESR erythrocyte sedimentation rate

Lauren et al. [30] found that D-dimer results vary significantly in different laboratories, even for the same sample. Thus, D-dimer as a PJI diagnosis criterion was refuted. In our study, D-dimer exhibited a low value in PJI diagnosis, and its numerical values remained high after ESR and CRP levels were decreased in the normal range. Moreover, these conventional coagulation biomarkers can only reflect quantitative changes in platelet and fibrinogen levels.

TEG can provide comprehensive coagulation status of our body and provide additional data compared with standard coagulation tests [21]. Many studies have

Table 4 Culture Results of PJI Patients

\begin{tabular}{lll}
\hline Classification & Strain & Number of patients \\
\hline G+ & S. aureus & 3 \\
& S. epidermidis & 2 \\
& S. dysgalactiae & 1 \\
& S. agalactiae & 1 \\
& S. intermedius & 1 \\
& Viridans Streptococci & 1 \\
& F. magna & 1 \\
& C. glutamicum & 1 \\
G- & E. coli & 2 \\
& Pseudomona aeruginosa & 1 \\
& Brucella & 1 \\
& Alcaligenes & 1 \\
Fungi & C. parapsilosis & 2 \\
Negative & Aspergillus flavus & 1 \\
Total & & 4 \\
\hline
\end{tabular}

G+ Gram-positive bacteria, G- Gram-negative bacteria compared TEG and conventional coagulation tests in many clinical fields. For example, Hani et al. [16] demonstrated that TEG provides more information about the haemostatic state of patients with cirrhosis than conventional coagulation tests. Furthermore, Luo et al. [31] reported that TEG may be a reliable alternative to conventional coagulation methods for diagnosing sepsisinduced coagulopathy.

To date, no study has compared the TEG's value with the three most used biomarkers (CRP, ESR, and Ddimer) in diagnosing PJI. In the present study, we highlight the value of ESR in diagnosing PJI, with AUC, optimal cut-off, sensitivity, and specificity for ESR of $0.953,34.0 \mathrm{~mm} / \mathrm{h}, 81.82$, and $94.87 \%$, respectively. Ddimer had the lowest AUC, and it remained high in PJI patients before the second-stage surgery. Although there were small differences in the numerical values of AUC, cut-off value, sensitivity, and specificity, our results are similar to those of several other studies [11, 12, 29]. Additionally, our study found that MA achieved a good diagnostic value with a specificity of $97.44 \%$. The combination of CRP/ESR with TEG parameters (K, Angle, MA, A30, TPI) achieved higher sensitivity and specificity than any individual marker, except for the two combinations (ESR + MA, ESR + A30). Thus, despite lower AUCs of these parameters than ESR, TEG remains a promising diagnostic test for PJI.

Performing the second-stage revision in proper timing is the key to boosting the success rate of PJI treatment. Hence, researchers continue exploring the optimal timing for reimplantation. In recent years, various indicators have been developed. Hoell et al. [32] reported that CRP was not a reliable parameter to exclude persistent infections. Tao Bian et al. [33] also concluded that ESR and CRP were of limited value in determining the 
Table 5 Serum Inflammatory and Fibrinolytic Markers in PJI Patients with Different Pathogens

\begin{tabular}{lllll}
\hline Markers & G+ $(\boldsymbol{n}=\mathbf{1 1})$ & G- $(\boldsymbol{n}=\mathbf{5})$ & Fungi $(\boldsymbol{n}=\mathbf{3})$ & Negative $(\boldsymbol{n}=\mathbf{4})$ \\
\hline CRP $(\mathrm{mg} / \mathrm{L})$ & $59.82 \pm 55.58$ & $45.40 \pm 83.25$ & $15.00 \pm 2.27$ & $30.33 \pm 15.73$ \\
ESR $(\mathrm{mm} / \mathrm{h})$ & $64.78 \pm 35.07$ & $55.20 \pm 25.33$ & $49.00 \pm 32.51$ & $55.75 \pm 16.26$ \\
D-dimer $(\mathrm{mg} / \mathrm{L})$ & $1.71 \pm 1.28$ & $1.33 \pm 0.89$ & $1.33 \pm 0.77$ & $2.52 \pm 1.64$ \\
K (min) & $1.46 \pm 1.08$ & $0.94 \pm 0.17$ & $1.33 \pm 0.06$ & $1.03 \pm 0.17$ \\
Angle $\left(^{\circ}\right)$ & $70.74 \pm 10.24$ & $75.74 \pm 1.68$ & $70.93 \pm 0.71$ & $74.83 \pm 2.70$ \\
MA (mm) & $70.63 \pm 7.47$ & $72.08 \pm 2.61$ & $68.73 \pm 2.79$ & $73.70 \pm 3.82$ \\
A30 $(\mathrm{mm})$ & $70.43 \pm 7.58$ & $71.58 \pm 3.27$ & $68.37 \pm 2.64$ & $72.98 \pm 4.69$ \\
TPI/(/sec) & $117.06 \pm 60.90$ & $141.20 \pm 36.25$ & $81.63 \pm 12.94$ & $166.45 \pm 51.92$ \\
\hline
\end{tabular}

$S D$ standard deviation, $C R P C$-reactive protein, ESR erythrocyte sedimentation rate, $K$ clotting time, Angle $a$-angle, $M A$ maximum amplitude, $A 30$ amplitude at 30 min, TPI thrombodynamic potential index

The values are expressed as the mean \pm SD

reimplantation timing by pooled analysis. Meanwhile, some studies report that coagulation-related biomarkers perform well in guiding reimplantation. Shahi et al. [14] highlighted D-dimer in determining the optimal timing of reimplantation. Moreover, Geng Bin et al. [10] reported fibrinogen as a useful tool for assessing infection outcomes after first-stage surgery. However, a small sample of both studies limited their credibility.

Furthermore, we found that ESR and MA were good indicators for determining the timing of reimplantation. Our study showed a $100 \%$ success rate of two-stage revision, which is higher than those reported in most published studies. Several reasons contribute to this result: 1 ) the follow-up time of 4 patients was shorter than 1 year, which may not be enough to judge infection control. 2) Although widely adopted by many researchers, the Delphi-based consensus is not the gold standard for evaluating the success of the two-stage revision, as it overlooks the functional outcomes of surgery. Therefore, our success rate would be lower when the functional outcome is considered.

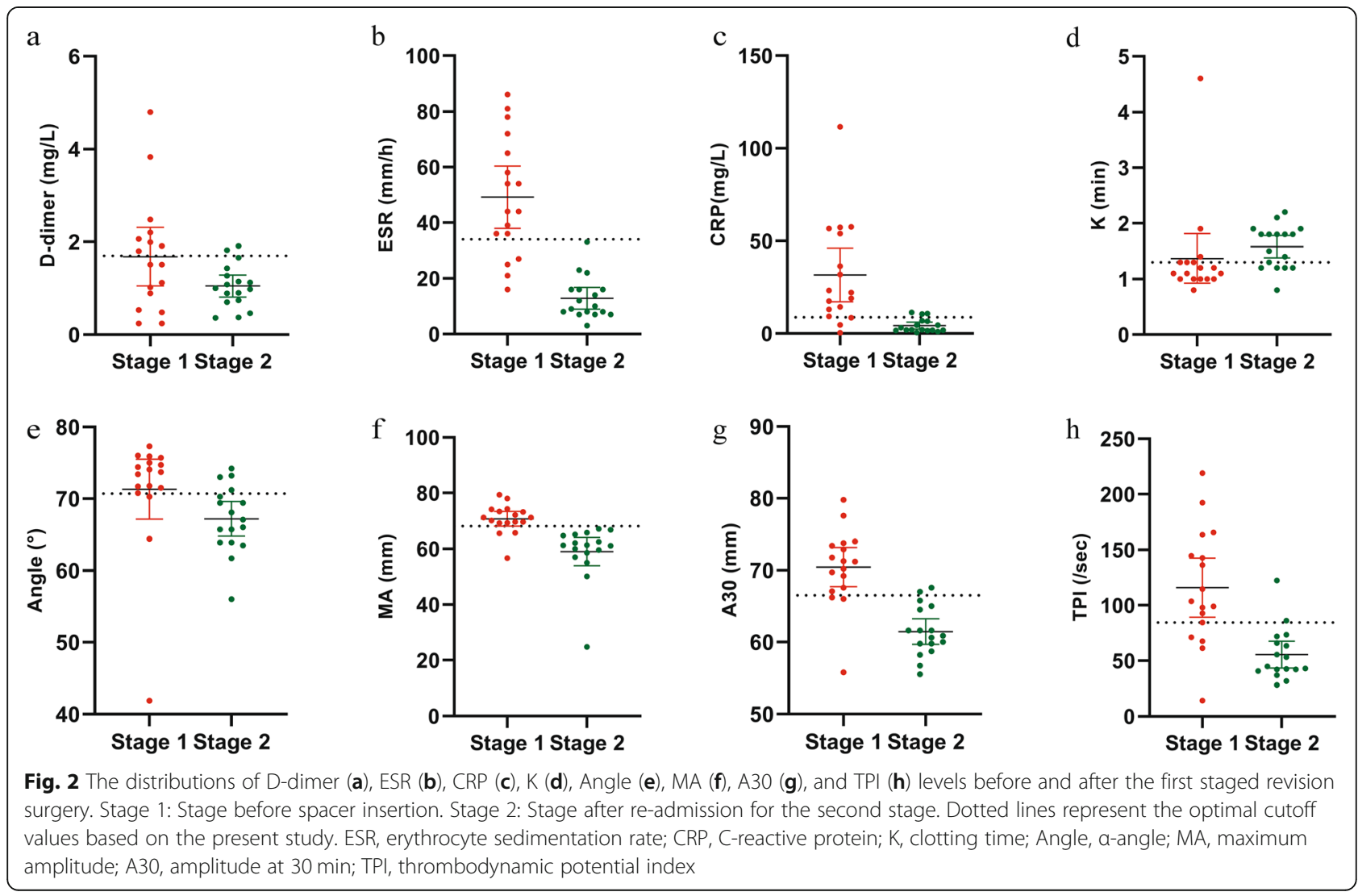


According to the results of this study, TEG has the following advantages. First, as a regular and routine serological test for coagulation, the TEG assay does not incur additional costs or suffering to patients. Second, the TEG parameters may be applied to differentiate PJI from aseptic loosening, particularly the combination of CRP and MA/A30. Finally, MA/A30 appears to be a valuable tool for assessing infection control after spacer insertion.

Our study has several limitations. First, the samples in this study were small; a larger sample size might have produced different results. Second, lower extremity Doppler ultrasound was routinely performed to exclude venous thromboembolism of the lower limb, which did not rule out clots in other parts of the patient's body. Moreover, we did not consider PJI patients' use of antibiotics before admission to our hospital. Furthermore, this study has some inherent biases due to its retrospective nature. Finally, we only checked the TEG of PJI patients before spacer insertion and before reimplantation, rather than checking them regularly. Hence, the changing trend of the biomarker levels in PJI patients was not clear.

\section{Conclusion}

This study reports 5 measured TEG parameters (K value, angle, MA, A30, TPI) that are statistically different between patients with PJI, with aseptic loosening, and those who were re-admitted for reimplantation in twostage arthroplasty. With high specificity, MA was considered a valuable biomarker in diagnosing PJI and assessing infection control after the first-stage surgery.

\begin{abstract}
Abbreviations
PJl: Periprosthetic joint infection; TEG: Thromboelastography; MSIS: Musculoskeletal Infection Society; CRP: C-reactive protein; ESR: Erythrocyte sedimentation rate; K: Clotting time; Angle: a-angle; MA: Maximum amplitude; A30: Amplitude at $30 \mathrm{~min}$; TPI: Thrombodynamic potential index; Fib: Fibrinogen; ROC: Receiver operating characteristic curves; AUCs: The areas under the curves; PPV: Positive predictive value; NPV: Negative predictive value; +LR: Positive likelihood ratio; -LR: Negative likelihood ratio; Cl: Confidence interval; TF: Tissue factor; ILs: Interleukins; TNFa: Tumor necrosis factor a; ICM: International Consensus Meeting
\end{abstract}

\section{Acknowledgments}

Not Applicable.

\section{Authors' contributions}

TY, the first author, participated in the design of the study, searched the data, performed the analysis, and drafted the manuscript. YW, the second author, participated in the design of the study, searched the data. SS, the corresponding author, participated in the design of the study and helped to draft the manuscript. All authors have read and approved the final manuscript.

\section{Authors' information}

SS, the corresponding author, is the chief physician of Department of Joint Surgery, Shandong Provincial Hospital.

\section{Funding}

This work was supported by the National Natural Science Foundation of China [grant number81972056]; the Natural Science Foundation of
Shandong Province of China [grant number ZR2018MH007]. The funding body played no role in the design of the study and collection, analysis, and interpretation of data and in writing the manuscript.

\section{Availability of data and materials}

The datasets used and/or analyzed during the current study are available from the corresponding author on reasonable request.

\section{Declarations}

Ethics approval and consent to participate

The study has acquired the ethics approval and the waivers of informed consent (NO. SWYX2020-185) from the Biomedical Research Ethics Committee of Shandong Provincial Hospital.

\section{Consent for publication \\ Not Applicable.}

\section{Competing interests}

The authors declare they have no financial interests.

Received: 20 January 2021 Accepted: 2 August 2021

Published online: 13 August 2021

\section{References}

1. Berns E, Barrett C, Gardezi M, Spake C, Glasser J, Antoci V, et al. Current clinical methods for detection of peri-prosthetic joint infection. Surg Infect. 2020;21(8):645-53. https://doi.org/10.1089/sur.2019.314.

2. Pannu TS, Villa JM, Patel PD, Riesgo AM, Barsoum WK, Higuera CA. The utility of serum D-dimerfor the diagnosis of periprosthetic joint infection in revision total hip and knee arthroplasty. J Arthroplast. 2020;35(6):1692-5. https://doi.org/10.1016/.arth.2020.01.034.

3. Tande AJ, Patel R. Prosthetic joint infection. Clin Microbiol Rev. 2014;27(2): 302-45. https://doi.org/10.1128/cmr.00111-13.

4. Xiong L, Li S, Dai M. Comparison of D-dimer with CRP and ESR for diagnosis of periprosthetic joint infection. J Orthop Surg Res. 2019;14(1):240. https:// doi.org/10.1186/s13018-019-1282-y.

5. Hu Q, Fu Y, Tang L. Serum D-dimeras a diagnostic index of PJI and retrospective analysis of etiology in patients with PJ. Clin Chim Acta. 2020; 506:67-71. https://doi.org/10.1016/j.cca.2020.03.023.

6. Qin L, Li F, Gong X, Wang J, Huang W, Hu N. Combined measurement of Ddimerand C-reactive protein levels: highly accurate for diagnosing chronic periprosthetic joint infection. J Arthroplast. 2020;35(1):229-34. https://doi. org/10.1016/j.arth.2019.08.012.

7. Corona PS, Vicente M, Carrera L, Rodríguez-Pardo D, Corró S. Current actual success rate of the two-stage exchange arthroplasty strategy in chronic hip and knee periprosthetic joint infection. Bone Joint J. 2020;102-b(12):1682-8. https://doi.org/10.1302/0301-620x.102b12.Bj-2020-0792.R1.

8. Fu J, Ni M, Li H, Li X, Chai W, Zhou Y, et al. The proper timing of secondstage revision in treating periprosthetic knee infection: reliable indicators and risk factors. J Orthop Surg Res. 2018;13(1):214. https://doi.org/10.1186/ s13018-018-0885-z.

9. Stambough JB, Curtin BM, Odum SM, Cross MB, Martin JR, Fehring TK. Does change in ESR and CRP guide the timing of two-stage arthroplasty reimplantation? Clin Orthop Relat R. 2019;477(2):364-71. https://doi.org/10.1 097/01.blo.0000533618.31937.45.

10. Bin G, Xinxin Y, Fan L, Shenghong W, Yayi X. Serum fibrinogen test performs well for the diagnosis of periprosthetic joint infection. Arthroplast. 2020;35(9):2607-12. https://doi.org/10.1016/j.arth.2020.04.081.

11. Wang Y, Li Y, Qiao L, Sun S. Comparison of a comprehensive set of fibrinolytic markers with C-reactive protein and erythrocyte sedimentation rate for the diagnosis of periprosthetic joint infection. J Arthroplast. 2020; 35(9):2613-8. https://doi.org/10.1016/j.arth.2020.04.096.

12. Wu H, Meng Z, Pan L, Liu H, Yang X, Yongping C. Plasma fibrinogen performs better than plasma D-dimerand fibrin degradation product in the diagnosis of Periprosthetic joint infection and determination of reimplantation timing. J Arthroplasty. 2020;35(8):2230-6. https://doi.org/10.1 016/j.arth.2020.03.055.

13. Xu H, Xie J, Yang J, Chen G, Huang Q, Pei F. Plasma fibrinogen and platelet count are referable tools for diagnosing periprosthetic joint infection: a 
single-center retrospective cohort study. J Arthroplast. 2020;35(5):1361-7. https://doi.org/10.1016/j.arth.2019.12.015.

14. Shahi A, Kheir MM, Tarabichi M, Hosseinzadeh HRS, Tan TL, Parvizi J. Serum D-dimer test is promising for the diagnosis of periprosthetic joint infection and timing of reimplantation. J Bone Joint Surg Am. 2017;99(17):1419-27. https://doi.org/10.2106/jbjs.16.01395.

15. Saraiva IE, Miranda PK, Freitas JN, Oliveira CR, Ataíde TL, Nobre V, et al. Thromboelastometric evaluation of sepsis associated coagulopathy: a cohort study. Thromb Res. 2016;147:124-5. https://doi.org/10.1016/j. thromres.2016.10.003.

16. Shamseddeen H, Patidar KR, Ghabril M, Desai AP, Nephew L, Kuehl S, et al. Features of blood clotting on thromboelastography in hospitalized patients with cirrhosis. Am J Med. 2020;133(12):1479-1487.e2. https://doi.org/10.101 6/j.amjmed.2020.04.029.

17. Zhou W, Zhou W, Bai J, Ma S, Liu Q, Ma X. TEG in the monitoring of coagulation changes in patients with sepsis and the clinical significance. Exp Ther Med. 2019;17(5):3373-82. https://doi.org/10.3892/etm.2019.7342.

18. Casado-Méndez M, Fernandez-Pacheco J, Arellano-Orden V, RodríguezMartorell FJ, Díaz-Martín A, Pastor de Las Heras Á, et al. Relationship of thromboelastography and conventional clotting test values with severe bleeding in critically ill patients with coagulopathy: a prospective study. Int J Lab Hematol. 2019;41(5):671-8. https://doi.org/10.1111/ijlh.13086.

19. Sokou R, Giallouros G, Konstantinidi A, Pantavou K, Nikolopoulos G, Bonovas $\mathrm{S}$, et al. Thromboelastometry for diagnosis of neonatal sepsis-associated coagulopathy: an observational study. Eur J Pediatr. 2018;177(3):355-62. https://doi.org/10.1007/s00431-017-3072-z.

20. Nates J, Aravindan N, Hirsch-Ginsberg C, Sizer K, Kee S, Nguyen A, et al. Critically ill cancer patients are not consistently hypercoagulable after craniotomy. Neurocrit Care. 2007;7(3):211-6. https://doi.org/10.1007/s12028007-0064-2.

21. Adamik B, Gozdzik W, Jakubczyk D, Welna M, Kübler A. Coagulation abnormalities identified by thromboelastometry in patients with severe sepsis: the relationship to endotoxemia and mortality. Blood Coagul Fibrin. 2017;28(2):163-70. https://doi.org/10.1097/mbc.0000000000000572.

22. Russell L, Haase N, Perner A. Prediction of bleeding by thromboelastography in ICU patients with haematological malignancy and severe sepsis. Blood Coagul Fibrin. 2018;29(8):683-8. https://doi.org/10.1097/ mbc.0000000000000777.

23. Parvizi J, Gehrke T. Definition of periprosthetic joint infection. J Arthroplast. 2014;29(7):1331. https://doi.org/10.1016/j.arth.2014.03.009.

24. Diaz-Ledezma C, Higuera C, Parvizi J. Success after treatment of periprosthetic joint infection: a Delphi-based international multidisciplinary consensus. Clin Orthop Relat Res. 2013;471(7):2374-82. https://doi.org/10.1 007/s11999-013-2866-1.

25. George J, Kwiecien G, Klika A, Ramanathan D, Bauer T, Barsoum W, et al. Are frozen sections and MSIS criteria reliable at the time of reimplantation of two-stage revision arthroplasty? Clin Orthop Relat Res. 2016;474(7):1619-26. https://doi.org/10.1007/s11999-015-4673-3.

26. Patel P, Walborn A, Rondina M, Fareed J, Hoppensteadt D. Markers of inflammation and infection in Sepsis and disseminated intravascular coagulation. Clin Appl Thromb-Hem. 2019;25(107602):9619843338. https:// doi.org/10.1177/1076029619843338.

27. Samuels JM, Moore HB, Moore EE. Coagulopathy in severe sepsis: interconnectivity of coagulation and the immune system. Surg Infect. 2018; 19(2):208-15. https://doi.org/10.1089/sur.2017.260.

28. Yan J, Xie K, Jiang X, Han X, Wang L, Yan M. D-dimer for diagnosis of periprosthetic joint infection: a meta-analysis. J Orthop Sci. 2020. https://doi. org/10.1016/j.jos.2020.09.015.

29. Li R, Shao HY, Hao LB, Yu BZ, Qu PF, Zhou YX, et al. Plasma fibrinogen exhibits better performance than plasma D-dimer in the diagnosis of periprosthetic joint infection: a multicenter retrospective study. J Bone Joint Surg Am. 2019;101 (7):613-9. https://doi.org/10.2106/jbjs.18.00624.

30. Pearson L, Moser K, Schmidt R. D-dimer varies widely across instrument platforms and is not a reliable indicator of periprosthetic joint infections. Arthroplast Today. 2020;6(4):686-8. https://doi.org/10.1016/j.artd.2020.07.014.

31. Luo C, Hu H, Gong J, Zhou Y, Chen Z, Cai S. The value of thromboelastography in the diagnosis of sepsis-induced coagulopathy. Clin Appl Thromb-Hem. 2020;26:1076029620951847. https://doi.org/10.1177/1 076029620951847

32. Hoell S, Moeller A, Gosheger G, Hardes J, Dieckmann R, Schulz D. Two-stage revision arthroplasty for periprosthetic joint infections: what is the value of cultures and white cell count in synovial fluid and CRP in serum before second stage reimplantation? Arch Orthop Trauma Surg. 2016;136(4):44752. https://doi.org/10.1007/s00402-015-2404-6.

33. Bian T, Shao H, Zhou Y, Huang Y, Song Y. Tests for predicting reimplantation success of two-stage revision for periprosthetic joint infection: a systematic review and meta-analysis. Orthop Traumatol-Sur. 2018;104(7):1115-23. https://doi.org/10.1016/j.otsr.2018.03.017.

\section{Publisher's Note}

Springer Nature remains neutral with regard to jurisdictional claims in published maps and institutional affiliations.
Ready to submit your research? Choose BMC and benefit from:

- fast, convenient online submission

- thorough peer review by experienced researchers in your field

- rapid publication on acceptance

- support for research data, including large and complex data types

- gold Open Access which fosters wider collaboration and increased citations

- maximum visibility for your research: over $100 \mathrm{M}$ website views per year

At BMC, research is always in progress.

Learn more biomedcentral.com/submissions 